\title{
Photoconverter with luminescent concentrator. Matrix material
}

\author{
M.R. Kulish ${ }^{1}$, V.P. Kostylyov ${ }^{1}$, A.V. Sachenko ${ }^{1}$, I.O. Sokolovskyi ${ }^{1}$, A.I. Shkrebtii ${ }^{2}$ \\ ${ }^{I}$ V. Lashkaryov Institute of Semiconductor Physics, NAS of Ukraine, \\ 45, prospect Nauky, 03680 Kyiv, Ukraine \\ ${ }^{2}$ University of Ontario Institute of Technology, \\ 2000 Simcoe St. N., Oshawa, Ontario, L1H 7K4, Canada
}

\begin{abstract}
Materials, promising for production of luminescent solar energy concentrators were considered. It is known that the silicon oxide matrix is transparent within the spectral range where Sun emits light. Up to date, the low-temperature sol-gel method for synthesizing $\mathrm{SiO}_{2}$ coatings with the simultaneous doping of the material with quantum dots (QDs) is developed. The transmission spectrum of borosilicate glasses (BK7) is narrower than that of $\mathrm{SiO}_{2}$. Typically, the doping of $\mathrm{BK} 7$ with the quantum dots of the group $\mathrm{A}^{\mathrm{II}} \mathrm{B}^{\mathrm{VI}}$ is carried out using the method of condensation at high temperatures, which results in a low value of the quantum yield of luminescence. Minimal losses of luminescent quanta through the leakage cone will be in the matrix of glass LASF35 022291.541, which refractive index is 2.022. In the research of the properties of photoconductors with a luminescent concentrator, the matrix is most often made of polymethylmethacrylate (PMMA). Its doping with QDs and dyes is well developed. The quantum yield of luminescence of luminophores when doping PMMA with dyes and QDs is close to unity. The magnitude of losses of luminescent quanta in matrices of glass, PMMA and silica has been estimated. Dependence of these losses in the wave range, which should be taken into account in the study of stacked fluorescent concentrators, has been analyzed.
\end{abstract}

Keywords: $\mathrm{SiO}_{2}$, borosilicate glass, polymethylmethacrylate, matrix, luminescent concentrator.

doi: https://doi.org/10.15407/spqeo22.01.80

PACS 88.40.jm, 88.40.jp

Manuscript received 15.01.19; revised version received 06.02.19; accepted for publication 20.02.19; published online 30.03.19.

\section{Introduction}

Generally, photoconverter with a fluorescent concentrator (FC) is transparent for a sunlight plate doped by a luminophore and combined with solar cells attached to its ends. The luminophores absorb light in a wide spectral range of sunlight quanta and emit luminescent quanta in a narrow spectral band. The luminophores emit quanta in a random direction. Typically, the refractive index of the plate exceeds the refractive index of the surrounding medium. Therefore, most of the quanta are transported to the ends of the plate due to full internal reflection and enter into the solar cell (SC), in which the energy of the luminescent quanta is converted into the electrical one. The rest of luminescent quanta leaves the plate and is lost. Since the area of end faces is much smaller than the area of the top face of the FC plate and the cost of this plate is low, it is expected that the photoconductors with FC will generate less expensive electricity $[1,2]$.
Photoconductors with a luminescent concentrator attract researchers by the fact that they are capable of converting into electrical energy not only the energy of direct incident light quanta but also the energy of the scattered light quanta [3]. It means that FCs do not need tracking systems for Sun's position. The large volume of the concentrator allows one to efficiently distribute the heat related with the relaxation losses. As a result, the solar cells will work at adequate temperature without using any cooling systems. An additional advantage of FCs is the possibility of their placement on the facades and roofs of buildings, which allows consuming the electricity produced by FC, simultaneously avoiding the solution of various technical problems when dealing with integration of photovoltaic systems into electrical networks [4].

The properties of material for luminescent concentrators define the efficiency of conversion of solar energy to electricity. To achieve maximum efficiency, the plate of the luminescent concentrator should have the following characteristics [5-9]: 
1) High transparency for sunlight in AM1 and AM1.5 conditions. Ideally, $100 \%$ optical transparency.

2) Almost ideal transparency in the emission spectrum of the fluorescence material.

3) Low scattering, especially in the spectral region of the highest response of the solar cell.

4) High refractive index. inertia.

5) High chemical resistance, perfect chemical

6) The ability to undergo plastic deformation without damage.

7) Great mechanical strength: the plate of the luminescent concentrator should not be deformed under its own weight.

8) Excellent weather resistance (fluctuations in temperature, wind, oxidation). Ideally for 20-30 years.

9) High photostability and durability for reaching the service life of more than 20-30 years.

10) Low cost required to minimize the cost of electricity.

11) Low density, preferably $<1 \mathrm{~g} / \mathrm{cm}^{3}$, so that the weight of the $100 \times 100 \times 1 \mathrm{~cm}$ plate should be $<10 \mathrm{~kg}$.

12) Zero toxicity.

13) The material of the matrix should be able to be doped with an appropriate luminophore while maintaining the quantum yield of luminescence close to unity.

For doping the matrix, three types of luminophores [7-9] are used: rare earth atoms and complexes, dyes, quantum dots (QDs). Of these, only QDs are capable to convert the energy of light quanta located in a wide spectral range into luminescence quanta in a narrow luminescent band. Usually, a quantum dot is a structure that consists of a core surrounded by one or more inorganic shells and one organic shell. Actually, in the core energy of wide spectral region quanta is transformed into a narrow luminescent band. Inorganic shells serve for passivation of dangling bonds on the surface of the core, providing a high quantum yield of QD luminescence. The organic shell prevents agglomeration of QDs, allowing to get the maximum possible concentration of QDs in the matrix. The need to preserve the organic shell means that the maximum temperature of the technological process of making a plate of doped QDs should not exceed the temperature of organic shell destruction.

\section{Matrix material}

The material of the matrix should be transparent for AM1.5 sunlight (Fig. 1, curve 4), which spectrum is within the range $0.25 \ldots 2.5 \mu \mathrm{m}[5,10]$. In principle, any material with a low absorption coefficient, which is transparent to AM1.5 light, can serve as a matrix [10]. Such materials may include inorganic materials (silica or glass of various types) and organic materials (various types of plastics). From the economic viewpoint, it is preferable to use silica glass of BK7 type, polymethylmethacrylate (PMMA).

\subsection{Silica}

According to [5-9, 13-15], fused silica transparent for AM1.5 sunlight (Fig. 1, curve 2) has a low content of inclusions, high uniformity of the refractive index, low absorption coefficient in the $0.35 \ldots 2.5 \mu \mathrm{m}$ spectral region (Fig. 1, curve 2). The refractive index in the entire spectral region is close to 1.5 (Fig. 2, curve 2).

Quantum dots can be injected into silica by using implantation of various atoms with subsequent annealing. For example, the authors of [20] reported formation of $\mathrm{ZnO}$ quantum dots in silica by using implantation of $\mathrm{Zn}$ and $\mathrm{F}$ ions with further annealing. Optical absorption and photoluminescence spectra measurements, electron microscopy and X-ray diffraction studies indicate that $\mathrm{ZnO}$ QDs are formed after annealing in air or in vacuum at temperatures above $500{ }^{\circ} \mathrm{C}$. Atomic force microscopy researches show a relatively smooth surface of annealed specimens, indicating that only $\mathrm{Zn}$ atoms are evaporated from the surface. Formation of $\mathrm{ZnO}$ QDs during thermal annealing can be explained by the direct oxidation of $\mathrm{Zn}$ nanoparticles by substrate oxygen molecules formed during implantation of $\mathrm{F}$ ions. The quality of $\mathrm{ZnO}$ QDs increases with increasing the annealing temperature.

The sol-gel method for the synthesis of CdS, CdSe, and CdTe QDs coated with an organic shell in the silicon matrix [21] has already been developed. This method allows obtaining monolithic silica with InP/ZnS (InP nucleus, $\mathrm{ZnS}$ - shell) quantum dots [22]. The quantum yield of $\mathrm{InP} / \mathrm{ZnS}$ quantum dots luminescence reached $21.7 \%$. With continuous excitation by using 400-nm light, the intensity of InP/ZnS QDs photoluminescence after 180 minutes was more than $90 \%$ of the initial value.

\subsection{Borosilicate glass (BK7)}

BK7 glass is one of the most common borosilicate glasses used for visible and near-infrared optics. Its high homogeneity, low content of bubbles and inclusions, the simplicity of manufacturing technology makes it suitable for preparation of FC matrix. BK7 demonstrates good scratch resistance. The transparency range for $\mathrm{BK} 7$ is $0.38 \ldots 2.1 \mu \mathrm{m}$ (Fig. 1, curve 3) [23]. The refractive index in the visible and near-infrared region is close to 1.5 (Fig. 2, curve 3).

To synthesize CdS QDs [21], CdS and elemental sulfur with amount approximately equal to one weight percent were added into a glass charge $\left(\mathrm{SiO}_{2} \approx 63 \ldots 70 \%\right.$, $\mathrm{B}_{2} \mathrm{O}_{3} \approx 3 \ldots 10 \%, \mathrm{ZnO} \approx 10 \ldots 15 \%, \mathrm{~K}_{2} \mathrm{O} \approx 10 \ldots 15 \%$, $\left.\mathrm{Na}_{2} \mathrm{O} \approx 10 \ldots 15 \%\right)$. This glass was melted at the temperatures $1200 \ldots 1400{ }^{\circ} \mathrm{C}$. At these temperatures, $\mathrm{Cd}$ and $\mathrm{S}$ are in the atomic-molecular dispersion state, which is sustained during rapid cooling of the melt to room temperature. The resulting solid solution of the semiconductor in the glass matrix is in a supersaturated state. Glass heating at $540{ }^{\circ} \mathrm{C}$ is accompanied by a diffuse decomposition of a supersaturated solid solution with fluctuation formation of the semiconductor phase 


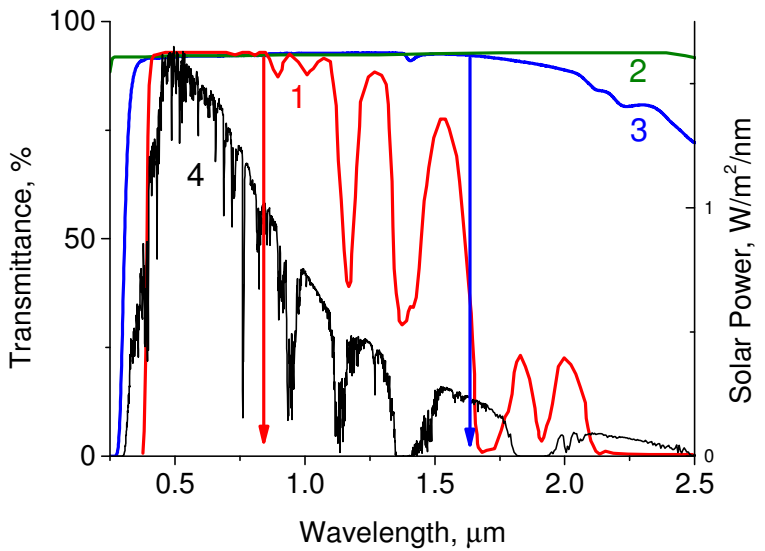

Fig. 1. Spectral distribution of the intensity of sunlight at the Earth surface (AM1.5G) (4) [11] and light transmission for polymethylmethacrylate (1) [12], silica of the type IR Grade (2) [15], borosilicate glass of the type N-BK7 (3) [16].

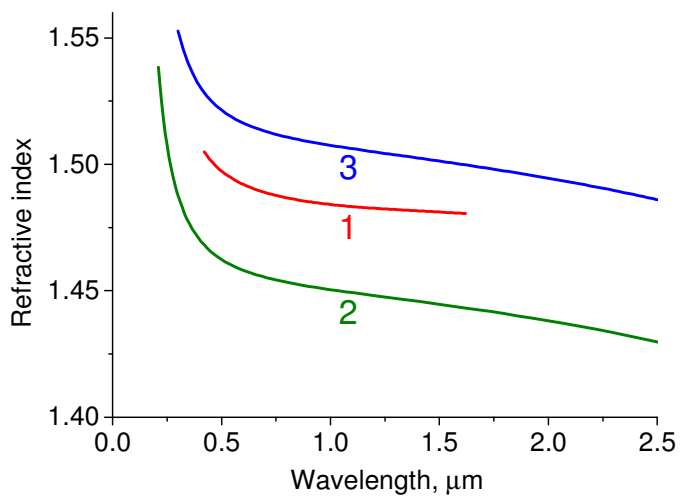

Fig. 2. Refractive index spectra for PMMA (1) [17], silica IR Grade (2) [18], glass BK7 (3) [19].

nuclei and a decrease in the degree of saturation. With the subsequent increase in the time of glass heating, the stage of condensation begins, when the increase in the size of nanocrystals occurs as a result of dissolution of small nanocrystals.

The inclusion of QDs into inorganic glasses allows combining the unique properties of QDs with good chemical, mechanical and thermal properties of these glasses. The inert nature of the glass matrix ensures photochemical stability and heat resistance of embedded QDs. However, the main problem of glasses containing $\mathrm{A}^{\mathrm{II}} \mathrm{B}^{\mathrm{VI}}$ group QDs is that defects on the interface between QDs and glass matrices quench exciton radiation. It is difficult to achieve a large quantum yield of luminescence with this technology of nanocrystal synthesis, since it is impossible to form inorganic and organic shells around nanocrystals.

\subsection{Organic plastics}

The majority of researches devoted to the study of the photoconductors with the luminescent concentrator properties is performed using the plates made of organic materials. In particular, the following types of polymers are promising for the FC matrix [24]: fluoropolymers (FP), ethylene backbone polymers (EBP), polyimides (PI). Their specifications are given in Table 1 [24].

The properties of the following plastics [25]: polymethylmethacrylate (PMMA), polyvinyl chloride (PVC), polyethylene terephthalate (PET) and polycarbonate (PC) were most often considered. Their transmission spectra are shown in Fig. 3 [25]. The data presented in Table 1 and Fig. 3 show that the transmission of these materials in the visible range differs little. Some differences in the transmission spectra are observed only in the ultraviolet and near-infrared spectral regions.

For the vast majority of plastics, the refractive index in the visible region of the spectrum is close to 1.5 (Table 1).

From all optically transparent plastics [26, 27], researchers prefer polymethylmethacrylate or PMMA $[12,17]$, since this material has better optical properties than any other organic polymer. PMMA is chemically stable, light and more transparent than glass (in the visible spectrum). Unfortunately, the PMMA transparency region is considerably narrower than the transparency region of silica and glass BK7. The degradation of the PMMA optical properties after 17 years of exposure to sunlight in the desert was insignificant [6].

PMMA is relatively inexpensive and synthesized easily. However, caution is required when manufacturing PMMA concentrators, since their optical properties depend heavily on production technology (for example,

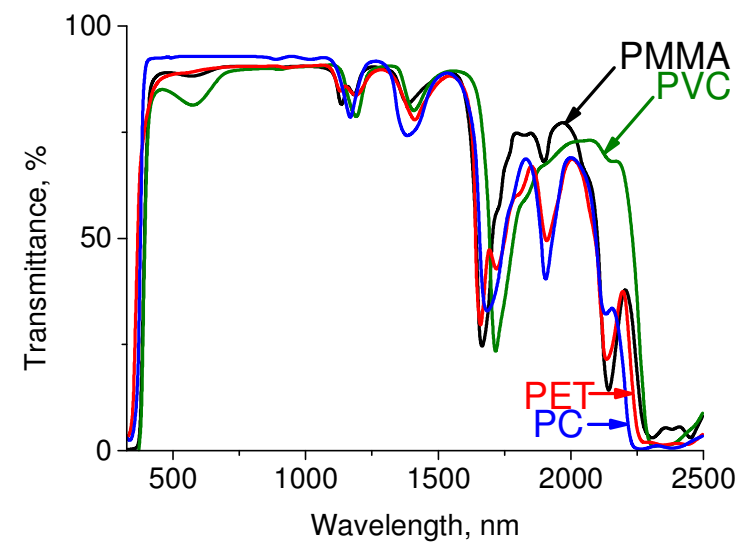

Fig. 3. Typical transmission (including Fresnel reflection losses) for 2-mm plastics [25]. 
Table 1. Organic plastics [24, 28].

\begin{tabular}{|c|c|c|c|c|c|c|c|c|}
\hline \multicolumn{3}{|c|}{ Fluoropolymers (FP) } & \multicolumn{3}{|c|}{ Ethylene backbone polymers (EBP) } & \multicolumn{3}{|c|}{ Polyimides (PI) } \\
\hline $\begin{array}{l}\text { Type } \\
\text { polymer }\end{array}$ & $\begin{array}{c}n \text { for } \\
\text { D-line } \\
(589.3 \mathrm{~nm})\end{array}$ & $\begin{array}{l}\text { Urbach } \\
\text { edge } \\
\text { position } \\
(\mathrm{nm})\end{array}$ & $\begin{array}{c}\text { Type } \\
\text { polymer }\end{array}$ & $\begin{array}{c}n \text { for } \\
\text { D-line } \\
(589.3 \mathrm{~nm})\end{array}$ & $\begin{array}{l}\text { Urbach } \\
\text { edge } \\
\text { position } \\
(\mathrm{nm})\end{array}$ & $\begin{array}{c}\text { Type } \\
\text { polymer }\end{array}$ & $\begin{array}{c}n \text { for } \\
\text { D-line } \\
(589.3 \mathrm{~nm})\end{array}$ & $\begin{array}{l}\text { Urbach } \\
\text { edge } \\
\text { position } \\
(\mathrm{nm})\end{array}$ \\
\hline $\begin{array}{l}\text { Tedlars } \\
\text { PVF } \\
\text { UT20BG3 }\end{array}$ & 1.474 & 454 & PV1400 & $\sim 1.49$ & 270 & Kapton ${ }^{\circledR} \mathrm{E}$ & $\sim 1.90$ & 763 \\
\hline $\begin{array}{l}\text { Teflons } \\
\text { ETFE }\end{array}$ & 1.398 & 314 & PV5200 & $\sim 1.48$ & $\sim 340$ & Kapton ${ }^{\circledR} \mathrm{H}$ & $\sim 1.82$ & 741 \\
\hline $\begin{array}{l}\text { PV1400 } \\
\text { EVA }\end{array}$ & 1.489 & 266 & PV5300 & $\sim 1.49$ & 346 & $\begin{array}{c}\text { Kapton }{ }^{\circledR} \\
\text { HN }\end{array}$ & $\sim 1.82$ & 731 \\
\hline $\begin{array}{l}\text { PV5200 } \\
\text { PVB }\end{array}$ & 1.480 & 366 & & & & & & \\
\hline $\begin{array}{l}\text { PV5300 } \\
\text { Ionomer }\end{array}$ & 1.487 & 346 & & & & & & \\
\hline Kaptons H & 1.824 & 741 & & & & & & \\
\hline $\begin{array}{l}\text { Kaptons } \\
\text { HN }\end{array}$ & 1.817 & 731 & & & & & & \\
\hline Kaptons E & 1.899 & 768 & & & & & & \\
\hline
\end{tabular}

injection molding, and compression). The study of the matrix manufacturing process [6] showed that the pouring of PMMA into a glass container and its drying to form a solid film is not optimal, since the solvent does not evaporate completely, inhomogeneities and dissipation losses arise in the PMMA plate.

The quantum yield of luminescence is close to unity for dyes injected into PMMA [8] and reaches $88.5 \%$ for $\mathrm{In}(\mathrm{Zn}) \mathrm{P} / \mathrm{ZnS}$ quantum dots [29]. For CdSe-ZnS/CdS/ZnS QDs, the quantum yield is close $100 \%$ (the quantum luminescence output already reaches 97\%) [30].

\section{Influence of the matrix material on the luminescent quanta losses through the leakage cone}

Of all types of luminophores, the most promising are QDs [8]. When QDs matrix is irradiated with sunlight, quantum dots absorb light quanta of the corresponding spectral interval and emit luminescent quanta in an arbitrary direction (Fig. 4). Usually, the matrix is surrounded by air. According to the Snell law, the reflection of light quanta from the boundary between the two media is described by the equation

$n_{m} \sin \theta_{\text {in }}=n_{\text {air }} \sin \theta_{\text {ref }}$,

where $n_{m}$ and $n_{\text {air }}$ are the refractive index of the matrix and air, $\theta_{i n}$ and $\theta_{\text {ref }}$ are the angle of incidence and that of light beam refraction, respectively.

When the angle of incidence increases, the refractive angle increases, and at a critical value of the angle of incidence $\theta_{i n}=\theta_{c}$, the refracted beam propagates along the surface of the matrix (Fig. 4), i.e., $\theta_{\text {ref }}=90^{\circ}$. In this case

$\theta_{c}(\lambda)=\arcsin \left(\frac{1}{n\left(\lambda_{\text {emit }}\right)}\right)$,

where $\lambda_{\text {emit }}$ is the wavelength of luminescent quanta. It means that all the luminescence quanta that fall onto the surface of matrix-air section at the angles $\theta_{i n}<\theta_{c}$ leave the matrix.

In most of materials suitable for production of the FC matrix, the refractive index is higher than 1.3 (see Fig. 2 and Table 2). The effect of refractive index on the magnitude of the critical angle is shown in Fig. 5. It is evident that, with $n_{m}$ increasing, the critical angle decreases and, therefore, reduction of luminescent quanta losses is expected.

If, however, the luminescent quanta fall onto the matrix-air boundary at the angles $\theta_{i n}>\theta_{c}$, due to total internal reflection, they have transported to the end faces of the matrix and proceed into the solar cells. In order to maximize the efficiency of photoconverters with luminescent concentrators, it is necessary to choose a matrix material with a maximum refractive index. In this case, $\theta_{c}$ will be minimal and, hence, the losses of quanta as a result of leakage through the cone will be minimal.

The fraction $P$ of luminescent quanta leaving the matrix is determined by the ratio of the solid angle of the cone of leakage $\Omega_{\text {cone }}=2 \pi\left(1-\cos \theta_{c}\right)$ to the $4 \pi$ steradian area sphere. 


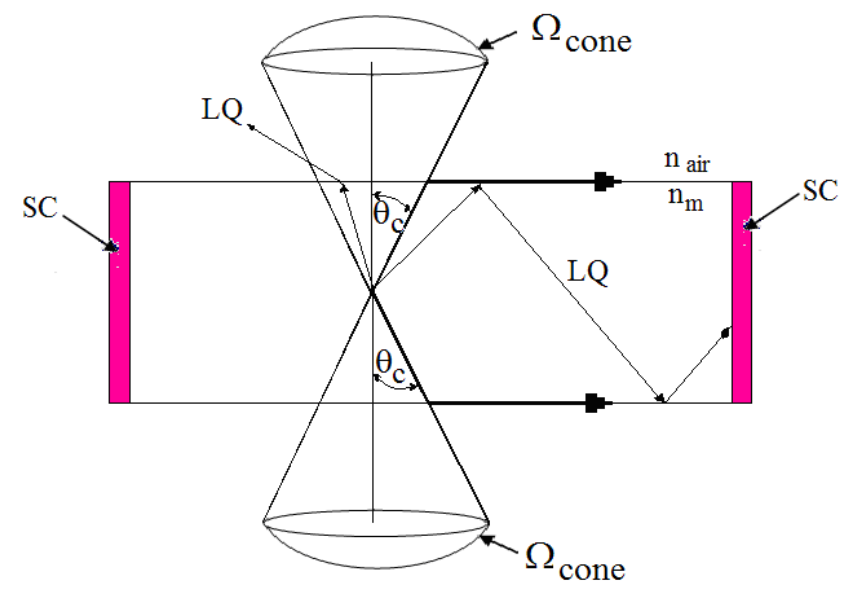

Fig. 4. Typical geometry of the FC matrix. The sunlight is absorbed by quantum dots (not shown in the figure). Luminescent quanta LQs can be emitted in an arbitrary direction. When they fall to the matrix-space separation at the angles $\theta<\theta_{c}$, they leave the matrix $\left(\theta_{c}\right.$ is the critical angle to which the refracted beam propagates along the matrix surface, $\Omega_{\text {cone }}$ is the solid angle of the cone of leakage). When luminescent quanta fall onto the matrix-air boundary $\left(n_{m}\right.$ and $n_{\text {air }}$ are the refractive index of the matrix and air, respectively) at the angles $\theta>\theta_{c}$, they are transported to the side face plate of the matrix through the full internal reflection and quanta fall to the entrance surface of the solar cell, where their energy is converted into electrical energy.

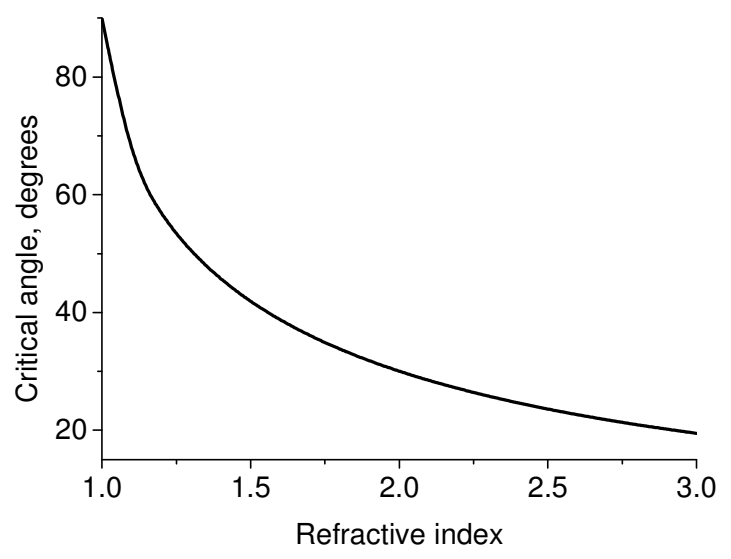

Fig. 5. The critical angle $v s$. the refractive index.
Therefore, following $[6,31]$

$$
P=\frac{2 \pi\left(1-\cos \theta_{c}\right)}{4 \pi}=\frac{1}{2}\left(1-\cos \theta_{c}\right) .
$$

Since the luminescent quanta flows through the input and rear surfaces of the matrix plate, then $P$ in the equation (3) should be multiplied by two. Hence, the fraction of the photons lost through the cones of leakage is described by the equation

$$
P=1-\cos \theta_{c} .
$$

Substituting $\theta_{c}$ values into the equation (4), we get that the fraction of lost photons is:

$$
P=1-\cos \theta_{c}=1-\cos \left(\arcsin \frac{1}{n_{m}}\right)=1-\sqrt{1-\frac{1}{n_{m}^{2}}} .
$$

Substituting the value of the refractive index in (5), the fraction $1-P$ of luminescent quanta (Fig. 6) captured in the matrix can be found. As expected, the fraction of photons captured in the matrix increases with the increase in the refractive index.

Following the given data, one can give the following recommendations for choosing the type of matrix for FC.

1) Only the matrix of silica is transparent for the entire range of AM1.5 and AM0 sunlight. It is necessary to improve technology of low-temperature synthesis of silica doped with quantum dots for their use. However, a relatively low refractive index value $\left(n_{m}=1.4584\right)$ for silica matrices should be taken into account. As a result, the critical angle $\theta_{c}$ of this material is large (Fig. 5), which leads to relatively large losses (the magnitude of losses reaches 27\%, see Fig. 8) of luminescent quanta through the cone of leakage.

2) Glass matrices are transparent for the main part of the solar spectrum. It is necessary to improve technology of injection for quantum dots with a high quantum yield of luminescence into the glass to use it.

The refractive index of the most common glass BK7 $n_{m}=1.4584$, which defines the relatively large value $\theta_{c}=41^{\circ}$. So, the losses of luminescent quanta through the

Table 2. Material parameters.

\begin{tabular}{|l|c|c|c|c|}
\hline Material & $n_{d}(\lambda=589.3 \mathrm{~nm})$ & Critical angle & Losses, \% & Trapped flux, \% \\
\hline Silica & 1.459 & 43.29 & 27.2 & 72.8 \\
\hline BK7 & 1.516 & 41.27 & 24.8 & 75.2 \\
\hline PMMA & 1.493 & 42.05 & 25.7 & 74.3 \\
\hline LASF35 022291.541 & 2.022 & 29.64 & 13.1 & 86.9 \\
\hline
\end{tabular}




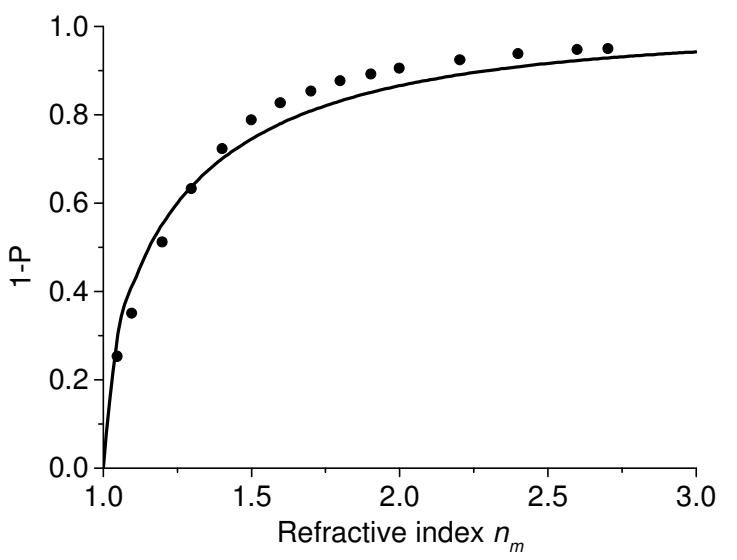

Fig. 6. Probability of capture of luminescent quanta in the matrix due to total internal reflection. The line - calculation by using the formula (5), points - calculation by simulating tracing of rays $[6,31]$.

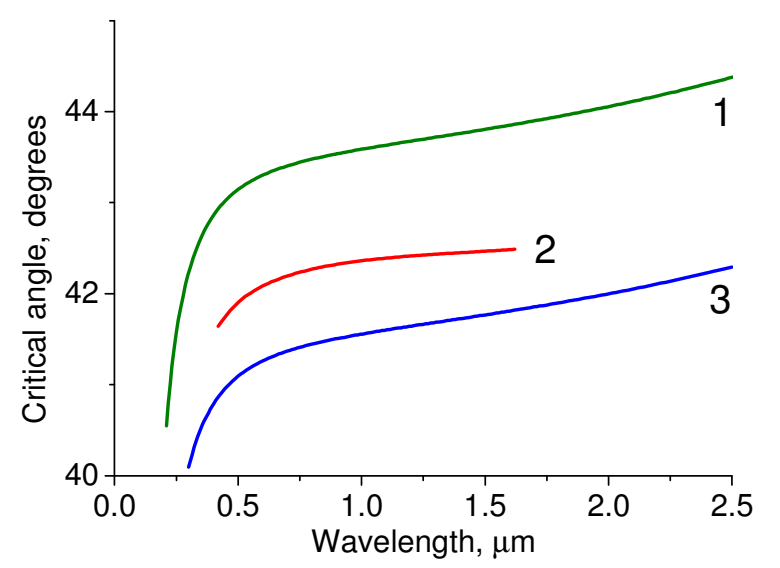

Fig. 7. Dependence of the critical angle on the wavelength for BK7 glass (1), PMMA (2), silica (3).

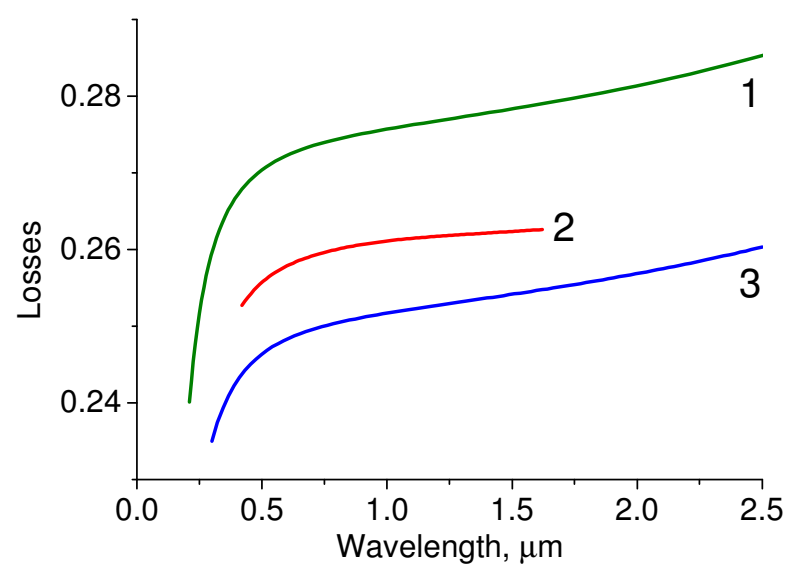

Fig. 8. Losses of luminescent quanta through the escape cone $v s$. the wavelength for BK7 glass (1), PMMA (2), silica (3). cone is significant $(P=24.8 \%)$ for this case. It is possible to significantly reduce these losses by using LASF35 022291.541 glass with $n_{m}=2.022$. For this glass, respectively, $\theta_{c}=29.64^{\circ}$ and the fraction of the luminescence quanta captured in the matrix can reach $86.9 \%$. However, we do not know about the use of this type of glass for production of the FC matrix.

3) PMMA matrices are widely used for the study of FC properties. The transmission spectrum for this matrix is limited by a range of approximately $400 \ldots 1000 \mathrm{~nm}$. The refractive index of this material $n_{m}=1.49$ defines a relatively large value of the critical angle $\theta_{c}=42^{\circ}$ and losses of luminescent quanta through the escape cone of $25.9 \%$.

4) To reduce the relaxation losses, FCs are put into the stack. The dispersion of the refractive index leads to a change in the losses of luminescent quanta through the cone. The dependences of the critical angle and losses of luminescent quanta through the escape cone are presented in Figs. 7 and 8. These parameters should be taken into account when analyzing the properties of FC in the stack.

\section{Conclusions}

The most complete use of solar energy in luminescent photoconverters is possible for the matrix of silicon oxide. Creation of this matrix requires the use of such methods as a low-temperature synthesis of $\mathrm{SiO}_{2}$ with simultaneous doping with quantum dots possessing high luminescence quantum yield.

The use of PMMA matrices doped with QDs is reasonable for developing the methods of light quanta losses reduction and optimization of the design of luminescent concentrators.

The use of a glass with high refractive index (for example, LASF35 022291.541 with $n_{m}=2.022$ ) gives the lowest critical angle and the minimal losses of luminescent quanta through the escape cone.

\section{References}

1. Yun-Seng Lim, Lo Chin Kim, Geok Bee Teh, Unsaturated polyester resin blended with MMA as potential host matrix for luminescent solar concentrator. Renewable Energy. 2012. 45. P. 156162. DOI: 10.1016/j.renene.2012.02.025.

2. Bende E.E., Slooff L.H., Burgers A.R., van Sагк W.G.J.H.M., Kenntdy M. Cost \& efficiency optimisation of the fluorescent solar-concentrator. The 23rd European photovoltaic solar energy conference, Valencia, Spain, September 1-5, 2008.

3. Mansour A.F., El-Shaarawy M.G., El-Bashir S.M., El-Mansy M.K., Hammam M. A qualitative study and field performance for a fluorescent solar collector. Polymer Testing. 2002. 21, No 3. P. $277 \mathrm{e} 81$. 
4. Meinardi F., Bruni F. \& Brovelli S. Luminescent solar concentrators for building-integrated photovoltaics. Nature Rev. Mater. 2017. 2. Article number 17072. DOI: 10.1038/natrevmats.2017.72.

5. Wilton S.R. Monte Carlo Ray-tracing Simulation for Optimizing Luminescent Solar Concentrators. A Thesis Degree of Master of Science. The Pennsylvania State University, The Graduate School College of Engineering Science, May 2012; https: //etda.libraries.psu.edu/files/final_submissions/7055.

6. Meyer T.J.J. Photon Transport in Fluorescent Solar Collectors. Thesis for the degree of Doctor of Philosophy, July 2009. University of Southampton faculty of engineering, science and mathematic school of engineering sciences materials research group; http://eprints.soton.ac.uk.

7. Gallagher S.J., Rowan B.C., Doran J., Norton B. Quantum dot solar concentrator: Device optimization using spectroscopic techniques. Solar Energy. 2007. 81. P. 540-547.

8. Kulish M.R., Kostylyov V.P., Sachenko A.V., Sokolovskyi I.O., Khomenko D.V., Shkrebtii A.I. Luminescent converter of solar light into electrical energy. Review. Semiconductor Physics, Quantum Electronics \& Optoelectronics. 2016. 19, No 3. P. 229-247.

9. Wilson L.R. Luminescent Solar Concentrators: A Study of Optical Properties, Reabsorption and Device Optimisation. Submitted for the degree of Doctor of Philosophy (Ph.D.) on completion of research conducted at the Department of Mechanical Engineering School of Engineering \& Physical Sciences. Heriot-Watt University Edinburgh, EH14 4AS United Kingdom. May 2010.

10. Voronkova Ye.M., Grechushnikov B.N., Distler G.I., Petrov I.P. Optical Materials for Infrared Technics. Moscow: Nauka, 1965 (in Russian).

11. ASTM G173-03 Tables: Extraterrestrial Spectrum, Terrestrial Global $37 \mathrm{deg}$ South Facing Tilt \& Direct Normal + Circumsolar; http://rredc.nrel.gov/solar/spectra/am1.5/

12. Measurement of Optical Characteristic of Plastic by UH4150 Spectrophotometer; https://www.hitachihightech.com/products/images/8414/uh4150_data1_ e.pdf.

13. http://www.technicalglass.com/fused_quartz_trans mission.html.

14. Fused Silica ( $\left.\mathrm{SiO}_{2}\right)$ IR Grade - International Crystal Laboratories;

http://www.internationalcrystal.net/optics_08.htm.

15. Fused Silica IR Grade $\left(\mathrm{SiO}_{2}\right)$; https://www.janis.com/Libraries/Window_Transmis sions/FusedSilicaIRGrade_SiO2_TransmissionCurv eDataSheet.sflb.ashx.

16. https://www.thorlabs.com/newgrouppage9.cfm?obj ectgroup_id=6973\&tabname=N-BK7.

17. Beadie G., Brindza M., Flynn R.A., Rosenberg A., and Shirk J.S. Refractive index measurements of poly(methyl methacrylate) (PMMA) from 0.4$1.6 \mu \mathrm{m}$. Appl. Opt. 2015. 54. P. F139-F143.
18. Malitson I.H. Interspecimen comparison of the refractive index of fused silica. J. Opt. Soc. Am. 1965. 55. P. 1205-1208.

19. SCHOTT Zemax catalog 2017-01-20b (http://www.schott.com).

20. Ren F., Zhang L.Y., Xiao X.H., Cai G.X., Fan L.X., Liao L. and Jiang C.Z. Controlling the growth of $\mathrm{ZnO}$ quantum dots embedded in silica by $\mathrm{Zn} / \mathrm{F}$ sequential ion implantation and subsequent annealing. Nanotechnology. 2008. 19, No 15, P. 155610. https://doi.org/10.1088/0957-4484/19/15/155610.

21. Fisher M. Optical Sensing with CdSe Quantum Dots in Condensed Phase Media. Electronic Theses, Treatises and Dissertations. 2009. Paper 4449.

22. Qiangbin Wang, Nora Iancu, and Dong-Kyun Seo. Preparation of Large Transparent Silica Monoliths with Embedded Photoluminescent CdSe@ZnS Core/Shell Quantum Dots. Chem. Mater. 2005. 17. P. 4762-4764.

23. Vargin V.V. Production of Colour Glass. MoscowLeningrad: Publ. House "Gizlegprom", 1940 (in Russian).

24. French R.H., Rodríguez-Parada J.M., Yang M.K. et al. Optical properties of polymeric materials for concentrator photovoltaic systems. Solar Energy Materials \& Solar Cells. 2011. 95. P. 2077-2086.

25. Measurement of Optical Characteristic of Plastic by UH4150 Spectrophotometer - An Example of High Throughput Measurements in the UV, Visible and Near-Infrared Regions; http://www.hitachihightech.com/products/images/8414/uh4150_data1_ e.pdf.

26. Knoll W. Optical Properties of Polymers, Materials Science and Technology. WILEY-VCH Verlag $\mathrm{GmbH} \& \mathrm{Co} \mathrm{KGaA}$;

https://onlinelibrary.wiley.com/doi/pdf/10.1002/978 3527603978.mst0143.

27. Stein R.S., and Finkelstein R.S. Optical properties of polymers. Ann. Rev. Phys. Chem. 1973. 24. P. 207-234; https://doi.org/10.1146/annurev. pc.24.100173.001231.

28. Li S., Lin M.M., Toprak M.S., Do Kyung Kim, and Muhammed M. Nanocomposites of polymer and inorganic nanoparticles for optical and magnetic applications. Nano Rev. 2010.

DOI: 1: 10.3402/nano.v1.0.5214.

29. Altintas Y., Talpur M.Y., and Mutlugün E. Cd-free quantum dot pellets for efficient white light generation. Opt. Exp. 2017. 25, No 23. P. 28371.

30. Samokhvalova P., Linkova P., Michel J., Molinari M. and Nabiev I. CdSe/ZnS photoluminescence quantum yield of $\mathrm{CdSe}-\mathrm{ZnS} / \mathrm{CdS} / \mathrm{ZnS}$ coremultishell quantum dots approaches $100 \%$ due to enhancement of charge carrier confinement. Proc. SPIE. 2014. 8955. P. 89550S-1; doi: $10.1117 / 12.2040196$.

31. Meyer T.J.J., Hlavaty J., Smith L. et al. Ray racing techniques applied to the modelling of fluorescent solar collectors. Proc. SPIE. 2009. 7211. P. $72110 \mathrm{~N}$. 


\section{Authors and CV}

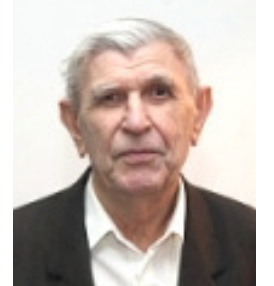

Kulish M.R. Doctor of Sciences in Physics and Mathematics, Leading Researcher at the Department of Optics and Spectroscopy, V. Lashkaryov Institute of Semiconductor Physics, NAS of Ukraine. The area of scientific interests of Dr. Kulish includes the investigation of optical and nonlinear optical properties of $3 \mathrm{D}$ and $0 \mathrm{D}$ semiconductor structures.

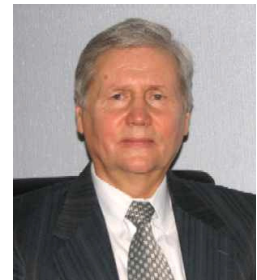

Kostylyov V.P. Doctor of Sciences in Physics and Mathematics, Head of the Laboratory of Physical and Technical Fundamentals of Semiconductor Photoenergetics, V. Lashkaryov Institute of Semiconductor Physics, NAS of Ukraine. The area of his scientific interests includes photovoltaic energy conversion physics in semiconductors and semiconductor photoconverters development.

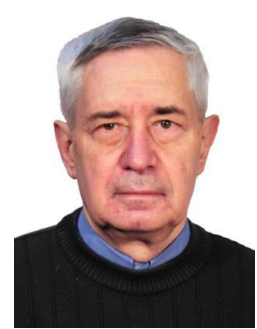

Sachenko A.V. Professor, Doctor of Sciences in Physics and Mathematics, Chief Researcher at the Department of Semiconductor Surface Physics and Photoelectricity, V. Lashkaryov Institute of Semiconductor Physics, NAS of Ukraine. The area of scientific interests of Prof. Sachenko includes physics of semiconductors and photovoltaics device simulation.

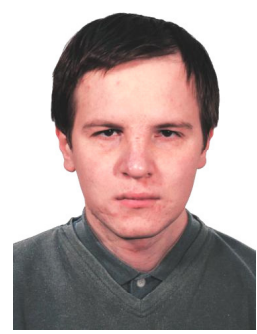

Sokolovskyi I.O. Ph.D. in Physics and Mathematics, Senior Scientist at the Department of Semiconductor Surface Physics and Photoelectricity, V. Lashkaryov Institute of Semiconductor Physics, NAS of Ukraine. The area of his scientific interests includes physics of photoconversion, analytical and numerical device simulation.

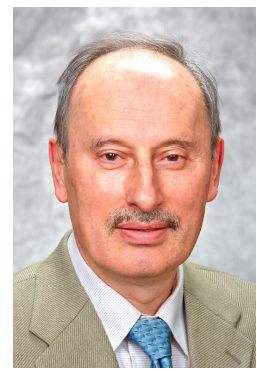

Shkrebtii A.I. Professor, Ph.D. in Physics and Mathematics, Professor at the University of Ontario, Institute of Technology. The area of scientific interests of Prof. Shkrebtii includes solid state physics, semiconductors and their surfaces as well as nanomaterials, nonlinear optical phenomena, electronic structural and dynamical properties of novel materials. 